\title{
EL CAMPAMENTO COMO PROGRAMA DIDÁCTICO: HACIA UNA PROPUESTA TEÓRICO-METODOLÓGICA PARA SU IMPLEMENTACIÓN EN LOS MUSEOS
}

THE CAMP AS A DIDACTIC PROGRAM: A THEORETICAL AND METHODOLOGICAL PROPOSAL TO THE IMPLEMENTATION IN MUSEUMS

\section{Volumen 11, Número 1}

pp. 1-33

Este número se publicó el 30 de abril de 2011

Manuel Enrique Luján Ferrer

Keilyn Rodríguez Sánchez

La revista está indexada en los directorios:

LATINDEX $, \underline{R E D A L Y C}, \underline{\text { IRESIE}}, \underline{\text { CLASE}}, \underline{\text { DIALNET, DOAJ, E-REVIST@S}}$

La revista está incluida en los sitios:

REDIE, RINACE, OEI, MAESTROTECA, PREAL, HUASCARAN, CLASCO 


\title{
EL CAMPAMENTO COMO PROGRAMA DIDÁCTICO: HACIA UNA PROPUESTA TEÓRICO-METODOLÓGICA PARA SU IMPLEMENTACIÓN EN LOS MUSEOS
}

THE CAMP AS A DIDACTIC PROGRAM: A THEORETICAL AND METHODOLOGICAL PROPOSAL TO THE IMPLEMENTATION IN MUSEUMS

\author{
Manuel Enrique Luján Ferrer ${ }^{1}$ \\ Keilyn Rodríguez Sánchez
}

\begin{abstract}
Resumen: Este artículo ofrece una definición de Campamento como programa didáctico, haciendo referencia a su origen, a su desarrollo y a su situación actual en la institución museal en el ámbito internacional. Además, esboza una reconstrucción histórica, analítica y crítica de los elementos teórico-metodológicos que fundamentan al Campamento en el contexto de la educación no formal y de la recreación educativa. Adicionalmente, identifica y describe diversas etapas de los Campamentos organizados según sus funciones y objetivos socio-educativos. Presenta los fundamentos conceptuales del Campamento como programa didáctico desde la educación no formal, donde los procesos de aprender haciendo, aprender jugando, aprender por descubrimiento, así como la colaboración y la cooperación son ejes esenciales. Señala aspectos centrales del liderazgo, de la formación de facilitadores y los principales aspectos de la administración de la educación no formal aplicados a un programa de Campamento, especialmente en el ámbito de los Museos. Lo anterior es complementado con la presentación de la experiencia del Museo de los Niños de Costa Rica: El Campamento Urbano Vacaciones Felices.
\end{abstract}

Palabras clave: EDUCACIÓN NO FORMAL, CAMPAMENTOS ORGANIZADOS, RECREACIÓN EDUCATIVA, PROGRAMAS EDUCATIVOS, MUSEOS, ADMINISTRACIÓN DE LA EDUCACIÓN NO FORMAL, EL CAMPAMENTO COMO PROGRAMA DIDÁCTICO, CAMPAMENTO URBANO EN EL MUSEO DE LOS NIÑOS.

\begin{abstract}
This article offers a definition of Camp as an educational program, referring to its origin, its development and current situation in the Museum as an institution in the international area. In addition, it outlines a historical reconstruction, analytical and critical of the theoretical and methodological elements that support the Camp in the context of non-formal education and educational recreation. Additionally, it identifies and describes various stages of the Camps organized by its functions and socio-educational goals. It presents the conceptual foundations of the Camp as an educational program from non-formal education in which the processes of learning by doing, learning by playing, learning by discovering, and collaboration and cooperation are key targets. It points out the main aspects of leadership, training leaders and fundamental aspects of managing non-formal education applied to a Camp program, especially in the area of Museums. As it was mention above, this is complemented by the presentation of the experience of the Children's Museum of Costa Rica: Urban Camp "Vacaciones Felices".
\end{abstract}

Keywords: NON-FORMAL EDUCATION, ORGANIZED CAMPS, EDUCATIONAL RECREATION, EDUCATIVE PROGRAMS, MUSEUMS, NON-FORMAL EDUCATION ADMINISTRATION, CAMPS AS DIDACTIC PROGRAMS, URBAN CAMP IN THE CHILDREN MUSEUM.

\footnotetext{
${ }^{1}$ Licenciatura en Antropología Social de la Universidad de Costa Rica y una Maestría en Tecnología Educativa de la Universidad Estatal a Distancia de Costa Rica y del Instituto Latinoamericano de Comunicación Educativa de México. Es profesor de la Universidad de Costa Rica. Fundador del programa de Campamento Urbano del Museo de los Niños de Costa Rica. Dirección electrónica: manuelenriquelujan@gmail.com

${ }^{2}$ Licenciatura en Antropología Social y otra en Ciencias de la Educación con énfasis en administración de programas de educación no formal, además tiene un doctorado en Educación, todos de la Universidad de Costa Rica. Fundadora del programa de Campamento Urbano del Museo de los Niños de Costa Rica. Es funcionaria del Ministerio de Cultura y Juventud, destacada al Centro Costarricense de Ciencia y Cultura (sede del Museo de los Niños). Dirección electrónica: keilynrosa@gmail.com
}

Artículo recibido: 31 de agosto, 2010

Aprobado: 7 de marzo. 2011 


\section{Presentación}

"Veo y me olvido,
oigo y recuerdo,
hago y comprendo".

Susana Gamboa

Este artículo ha surgido como una respuesta a la necesidad de dar a conocer los Campamentos como programas didácticos y a la responsabilidad que acompaña la experiencia acumulada por la autora y el autor en más de 25 años de trabajo en Campamentos, tanto en el ámbito de los Museos como en organizaciones religiosas y no gubernamentales.

Nuestro interés central es contribuir con las discusiones en torno al quehacer educativo no formal de los museos costarricenses y presentar las orientaciones teórico-metodológicas de las prácticas del campamento educativo.

En este sentido, el presente artículo consiste en una reflexión y propuesta teóricometodológica sobre los Campamentos como programas didácticos en el ámbito de la educación no formal y de la recreación educativa.

Con base en lo indicado, interesa destacar en el artículo la definición de Campamento como programa didáctico, el origen, los aspectos de su desarrollo en el tiempo, los fundamentos teórico-metodológicos, los procesos y fases que implica la administración de este programa educativo no formal. También, el vínculo entre museos y Campamentos, la relación entre recreación y educación y, por último, expondremos, brevemente, el caso del Campamento Urbano del Museo de los Niños: Vacaciones Felices.

\section{Concepto y evolución de los Campamentos organizados a nivel internacional}

Los primeros Campamentos organizados tienen su origen en la necesidad de los pobladores de las grandes ciudades por establecer un contacto directo con la naturaleza lejos de las comodidades que ofrece lo urbano; significó un volver a lo rústico; los campamentos son, en gran parte, el producto de una búsqueda de vivencias y actividades al aire libre.

En este sentido, Paris (2008) indica que en 1850 se hace referencia a la necesidad de cuidar la salud durante el tiempo vacacional. Así, se desarrolla todo un movimiento de turismo rural y el concepto de vacaciones de verano se pone de moda. Y para 1860, la promoción de las vacaciones se hace para que las personas acampen y disfruten de los simples placeres de 
la naturaleza; tiendas de campaña, pesca y caza son la moda durante este tiempo libre y de manera muy recreativa.

Inicialmente, el Campamento organizado se concibió como una forma de educación, que incluía un determinado tipo de vida en una comunidad cooperativa con predominio de actividades al aire libre. El primer país donde se da esta práctica es en los Estados Unidos de Norteamérica. Un impulso que provoca el surgimiento de los Campamentos, denominados Camp folclore (Watts, 2007) fue la creciente industrialización y la urbanización en los países desarrollados, que privaron a las personas de su sentido de relación con el mundo natural. Parker (2008) indica que el primer Campamento fue realizado por Frederick Williams Gunn, reconocido como el director del Campamento Gunnery en el año 1861.

Sin embargo, Paris (2008) señala que el Dr. Joseph Trimble Rothrocks, de North Mountain School of Physical Culture, en 1876 realizó un Campamento más completo que el de Gunn en el lago Garrrago. El Dr. Rothrocks se graduó en Harvard, era conservacionista, botánico y veterano de la Guerra Civil. Su formación y vivencias constituyeron parte de este Campamento. Un dato curioso es que el costo por campista fue de $\$ 200$ dólares estadounidenses por cuatro meses.

Ahora bien, todas estas experiencias nacidas en estas décadas están dirigidas, únicamente, hacia los niños y los adolescentes. Hasta en 1903 es cuando se inician los Campamentos para las niñas y las adolescentes planteados por movimientos cristianos protestantes, pero con condiciones diferentes por considerarlas "sexo débil" (Paris, 2008).

Este autor plantea que desde los inicios hasta finales del siglo XIX y a partir 1921, en Estados Unidos de Norteamérica cobra un gran auge el movimiento de Campamentos organizados, principalmente por el beneficio efectivo para la niñez y la juventud. Por ser una oportunidad educativa y recreativa, el campamento se constituye en una verdadera fuerza social.

Según Paris (2008) a partir de la década de los años veinte hay dos nuevas direcciones que asumen los Campamentos en Estados Unidos de Norteamérica, acordes con intereses de los padres y las madres de familia. La primera consiste en afinar las habilidades para el futuro profesional de sus hijos e hijas; la segunda se refiere a la utilización del Campamento como opción para niños y niñas que presentan problemas de personalidad o familiares. 
Así, los Campamentos de larga duración se transforman en un segundo hogar para muchas personas campistas. Lo anterior da lugar a que empiece a desarrollarse el concepto de Campamento temático.

Para 1980 en Estados Unidos de Norteamérica y Canadá se ofrecían 1.400 Campamentos particulares que anualmente atendían a una población infantil y juvenil de 200.000 personas. Sumados a otras organizaciones, entre ellas la Asociación Cristiana de Jóvenes, Boy Scouts de América, Comunidades Israelitas, Camp Fire Girls, llegaron a estimar 5000 Campamentos, donde atendían anualmente a más de 3.000 .000 de personas y durante la temporada de verano a unas 4.000.000 (Vigo, 2005).

Debido al éxito anterior, en Estados Unidos de Norteamérica es posible encontrar guías impresas de Campamentos de verano como la de Thompson Learning Corporation (2009) que cuenta con 26 ediciones y detalla más de 3.000 Campamentos para niños, niñas y adolescentes.

A manera de síntesis en el Cuadro 1 se presenta una cronología del origen de los Campamentos pioneros, organizados y educativos en el ámbito internacional, según país y año, organización fundadora, tipo de Campamento y algunas observaciones relacionadas con el tipo de participantes, los ejes temáticos, las actividades y los objetivos. 
Cuadro No. 1.

Cronología, a nivel internacional, del origen de los Campamentos pioneros.

\begin{tabular}{|c|c|c|c|}
\hline PAÍS Y AÑO & $\begin{array}{l}\text { ORGANIZACIÓN } \\
\text { FUNDADORA }\end{array}$ & $\begin{array}{c}\text { TIPO DE } \\
\text { CAMPAMENTO }\end{array}$ & OBSERVACIONES \\
\hline $\begin{array}{l}\text { Estados } \\
\text { Unidos de } \\
\text { América } \\
1861\end{array}$ & $\begin{array}{l}\text { Gunnery School } \\
\text { Frederick Williams } \\
\text { Gunn, Director. }\end{array}$ & $\begin{array}{l}\text { Programa de } \\
\text { verano estudiantil } \\
\text { al aire libre. }\end{array}$ & $\begin{array}{l}\text { Participan estudiantes de educación } \\
\text { secundaria. }\end{array}$ \\
\hline $\begin{array}{l}\text { Estados } \\
\text { Unidos } \\
\text { América } \\
1876\end{array}$ & $\begin{array}{l}\text { Escuela Nacional de } \\
\text { Educación Física. } \\
\text { Dr. Rothrocks. }\end{array}$ & $\begin{array}{ll}\text { Campamento } & \\
\text { enfocado } & a \\
\text { recreación } & y \\
\text { deporte. } & \\
\end{array}$ & $\begin{array}{l}\text { Énfasis en la práctica de las } \\
\text { competencias deportivas } \\
\text { acondicionamiento físico. }\end{array}$ \\
\hline $\begin{array}{l}\text { Estados } \\
\text { Unidos de } \\
\text { América } \\
1880\end{array}$ & $\begin{array}{l}\text { Asociación Cristiana } \\
\text { de Jóvenes } \\
\text { (YMCA) }\end{array}$ & $\begin{array}{lr}\text { Giras } & 0 \\
\text { "vagabundeos" en } \\
\text { verano al aire libre. }\end{array}$ & $\begin{array}{l}\text { Función recreativa y de contacto } \\
\text { con la naturaleza. }\end{array}$ \\
\hline $\begin{array}{l}\text { Estados } \\
\text { Unidos } \\
\text { América } \\
1885\end{array}$ & $\begin{array}{l}\text { Asociación de } \\
\text { Newbusgh } \\
\text { Summer F. Dudley, } \\
\text { Director. }\end{array}$ & $\begin{array}{l}\text { Rural a orillas del } \\
\text { lago Orange, Sur } \\
\text { de California y del } \\
\text { lago Champlain, } \\
\text { frontera } \quad \text { con } \\
\text { Canadá. }\end{array}$ & $\begin{array}{l}\text { Dudley influye poderosamente en la } \\
\text { difusión de este tipo de } \\
\text { Campamentos organizados en } \\
\text { Canadá y los Estados Unidos de } \\
\text { América. }\end{array}$ \\
\hline $\begin{array}{l}\text { Estados } \\
\text { Unidos de } \\
\text { América } \\
1903\end{array}$ & $\begin{array}{lr}\text { Asociación Cristiana } \\
\text { de Jóvenes. Luther } \\
\text { Gulick, promovió la } \\
\text { formación de } 2 \\
\text { grupos } \\
\text { Campamentos: los } \\
\text { "boy scouts of } \\
\text { America" y su } \\
\text { equivalente femeni- } \\
\text { no "Camp fire girls" }\end{array}$ & $\begin{array}{l}\text { Rural al aire libre, } \\
\text { recreativo y de } \\
\text { convivencia con el } \\
\text { ambiente natural y } \\
\text { rústico. }\end{array}$ & $\begin{array}{l}\text { Primer Campamento para mujeres. } \\
\text { Origen de las "Camp fire girls". }\end{array}$ \\
\hline $\begin{array}{l}\text { Argentina } \\
1903\end{array}$ & $\begin{array}{l}\text { Asociación Cristiana } \\
\text { de Jóvenes, Director } \\
\text { Bertrand Shuman. }\end{array}$ & $\begin{array}{l}\text { Rural educativo y } \\
\text { recreativo para } \\
\text { jóvenes. }\end{array}$ & Reducido número de campistas. \\
\hline $\begin{array}{l}\text { Canadá } \\
1921\end{array}$ & $\begin{array}{l}\text { Comité Nacional } \\
\text { Canadiense }\end{array}$ & $\begin{array}{l}\text { Rural educativo y } \\
\text { recreativo para } \\
\text { niños, se llamó } \\
\text { "Ahmek" en el lago } \\
\text { Canoe. }\end{array}$ & $\begin{array}{l}\text { Ideas principales: } \\
\text { 1. No proporcionar nada que pueda } \\
\text { quitar a los campistas la oportunidad } \\
\text { de crear. } \\
\text { 2. Experimentar sobre la influencia } \\
\text { del Campamento en el desarrollo } \\
\text { del carácter. }\end{array}$ \\
\hline
\end{tabular}

Fuente: Elaboración propia con base en planteamientos de Vigo (2005).

Según se puede observar en el Cuadro No. 1 Estados Unidos, Canadá y Argentina son los países que estuvieron a la vanguardia en gestar Campamentos; al mismo tiempo, si bien tomó un par de décadas, muy temprano incorporar se logra a las mujeres en el movimiento campista. 
Es importante destacar que la evolución de los Campamentos organizados se puede sistematizar en varias etapas de acuerdo con las funciones, los objetivos, las actividades y las tendencias metodológicas. Las experiencias particulares y de aprendizaje se transforman o cambian en el transcurso del tiempo, y se ajustan o mejoran en cuanto a sus proyecciones educativas, recreativas y de convivencia social.

Por lo tanto, el tipo de programa y de actividades incorporadas para organizar e implementar los Campamentos ha cambiado y se ha ajustado en función del mejoramiento de ejecución de los programas, la orientación hacia los fines educativos y de sana convivencia social. Esto de acuerdo con los objetivos establecidos por sus gestores.

En este sentido, la formación de líderes incluye aspectos de la programación del Campamento, la incorporación de temáticas sanitarias, de seguridad, de planificación, de organización, de ejecución y de evaluación que provoque su avance en la historia de los mismos.

Siguiendo el planteamiento de Vigo (2005) a continuación sistematizaremos en el Cuadro No. 2 las etapas de los Campamentos organizados según sus funciones y objetivos indicando las características principales para cada etapa.

Cuadro No. 2.

Etapas de los Campamentos organizados según sus funciones y objetivos socioeducativos.

\begin{tabular}{|c|c|c|}
\hline ETAPAS & FUNCIONES OBJETIVOS & OBSERVACIONES \\
\hline $\begin{array}{c}\text { RECREATIVA } \\
\text { Campamentos familiares } \\
\text { según sexo y edad en } \\
\text { zonas rústicas y } \\
\text { naturales. } \\
\text { Finales de siglo XIX } \\
\text { hasta la década de los } \\
\text { años } 20 \text { del siglo XX. }\end{array}$ & $\begin{array}{l}\text { Experiencia al aire libre y } \\
\text { contacto con la naturaleza. } \\
\text { Contrarrestar los efectos de } \\
\text { la industrialización en la } \\
\text { cultura. }\end{array}$ & $\begin{array}{l}\text { Etapa inicial de los } \\
\text { Campamentos. } \\
\text { Aspectos positivos: el valor de la } \\
\text { experiencia al aire libre como } \\
\text { liberación de la mecanización. } \\
\text { El reconocimiento de que valores } \\
\text { tales como la salud, el desarrollo } \\
\text { del carácter y el ajuste social } \\
\text { están presentes en la } \\
\text { experiencia, aunque hoy se } \\
\text { difiera del método aplicado para } \\
\text { alcanzarlos. } \\
\text { La idea de que el trabajo y el } \\
\text { esfuerzo son plasmadores de la } \\
\text { personalidad. }\end{array}$ \\
\hline
\end{tabular}




\begin{tabular}{|c|c|c|}
\hline & & $\begin{array}{l}\text { Aspectos débiles del período: La } \\
\text { intensidad del ritmo de vida hacía } \\
\text { que el Campamento fuera } \\
\text { fatigoso en demasía. Al } \\
\text { programar y ejecutar } \\
\text { directamente el staff, se tenía } \\
\text { muy poco en cuenta el } \\
\text { pensamiento del campista como } \\
\text { persona. }\end{array}$ \\
\hline $\begin{array}{c}\text { EDUCACIONAL } \\
\text { Campamentos y } \\
\text { Campamentos } \\
\text { temáticos, desarrollo de } \\
\text { infraestructura } \\
\text { específica. } \\
\text { Inicia en los años } \\
\text { posteriores a la Primera } \\
\text { Guerra Mundial y se } \\
\text { extiende hasta las } \\
\text { décadas de los años } 30 \text { y } \\
40 .\end{array}$ & $\begin{array}{l}\text { Esfuerzo sistemático para } \\
\text { identificar, conocer y proveer } \\
\text { las condiciones necesarias } \\
\text { para el logro de los objetivos } \\
\text { del Campamento en el } \\
\text { desarrollo de la salud, la } \\
\text { personalidad y el ajuste } \\
\text { social. } \\
\text { (Lo anterior forzó a formular } \\
\text { los objetivos de una manera } \\
\text { real y específica) } \\
\text { Programar las actividades } \\
\text { como proceso total de vida } \\
\text { en la comunidad del } \\
\text { Campamento y eliminar la } \\
\text { improvisación en el desarrollo } \\
\text { del programa. }\end{array}$ & $\begin{array}{l}\text { El personal de los Campamentos } \\
\text { empezó a ser seleccionado, } \\
\text { entrenado y supervisado, } \\
\text { teniendo en cuenta su rol } \\
\text { educativo. } \\
\text { Aspectos positivos del período: } \\
\text {-El fundamento científico de la } \\
\text { acción del Campamento. } \\
\text {-La formulación de propósitos y } \\
\text { objetivos concretos. } \\
\text {-La consideración del campista } \\
\text { como persona (toma en cuenta } \\
\text { sus deseos, intereses y } \\
\text { necesidades) } \\
\text {-La definición de la vocación } \\
\text { educacional del Campamento. } \\
\text { Aspectos débiles del período: } \\
\text {-Desarrollo exagerado de las } \\
\text { condiciones de infraestructura } \\
\text { limita las positivas enseñanzas de } \\
\text { la vida rústica o natural. } \\
\text {-La excesiva libertad dada al } \\
\text { campista producía desorientación } \\
\text { y desvió de los objetivos } \\
\text { educacionales propuestos. }\end{array}$ \\
\hline $\begin{array}{c}\text { Day Camp, Campamento } \\
\text { y Campamento Urbano } \\
\text { ORIENTACIÓN Y } \\
\text { RESPONSABILIDAD } \\
\text { SOCIAL } \\
\text { Desde la década de los } \\
\text { años } 50 \text { hasta el } \\
\text { presente. }\end{array}$ & $\begin{array}{l}\text { Formular, sistematizar y } \\
\text { aplicar estándares } \\
\text { normativos en el trabajo de } \\
\text { Campamentos, en lo } \\
\text { referente a la administración, } \\
\text { la organización, la salud, la } \\
\text { seguridad, el programa y el } \\
\text { personal. } \\
\begin{array}{l}\text { Arraigar los fundamentos } \\
\text { democráticos entre las } \\
\text { personas campistas. }\end{array}\end{array}$ & $\begin{array}{l}\text { El personal de Campamentos } \\
\text { acepta la actual tendencia del } \\
\text { trabajo en equipo y la necesidad } \\
\text { de utilizar la oportunidad } \\
\text { educacional del Campamento } \\
\text { para servir a la comunidad. } \\
\text { Re-orientación del programa, de } \\
\text { los objetivos y del liderazgo en } \\
\text { los Campamentos de verano. }\end{array}$ \\
\hline
\end{tabular}

Fuente: Elaboración propia con base en Vigo (2005). 
Según notamos en el cuadro anterior la profesionalización del quehacer del Campamento aumenta conforme pasa el tiempo y se atienden necesidades recreativas y educativas del momento en que se gesta.

Así las cosas, los Campamentos responden a necesidades sociales que Vigo (2005) resume así:

a) Uso inteligente del tiempo libre para lograr un equilibrio del binomio estudio-trabajo y recreación-tiempo libre.

b) Fomento de espacios de creatividad y esparcimiento al aire libre y, agregamos, en el medio urbano.

c) Promoción de la formación integral de la personalidad mediante una amplia variedad de vivencias.

d) Experiencias educativas alternativas y no formales para promover aprendizajes no tradicionales y participativos.

e) Experiencias fuera del control familiar (Por ejemplo, el caso del Campamento Urbano de Vacaciones Felices del Museo de los Niños)

f) Formación con otro grupo fuera del núcleo familiar.

g) Experiencias de vida cooperativa.

\subsection{Fundamentos conceptuales de los Campamentos organizados}

Dada la gran diversidad de Campamentos que existe, resulta complejo definir esta modalidad educativa. De hecho, no se registró en la bibliografía consultada, una definición que incluyera tanto a los Campamentos con hospedaje, como a los Campamentos Urbanos o Day Camp. Sin embargo, proponemos la siguiente definición:

el Campamento es un programa didáctico de la educación no formal, diseñado para el uso inteligente del tiempo libre, responde a intereses temáticos diversos según el ámbito cultural y el contexto socio económico en el cual se gesta. Su diseño supone no solo un proceso de planificación didáctica, sino también un proceso de administración educativa no formal, con sus fases de organización, ejecución y evaluación del personal involucrado, el presupuesto, objetivos, metodologías, contenidos, actividades, recursos y espacios físicos (elaboración propia).

Un Campamento es diseñado para que se desarrolle durante el tiempo libre de sus campistas, por lo que se hace referencia al concepto de recreación educativa. Fermoso (1997) 
llama a esto pedagogía del ocio y del tiempo libre, como una rama de la Educación No Formal. Esta pedagogía es originada en España y Francia por las décadas del 60 y del 70, donde se dio un gran movimiento a favor de las actividades organizadas extraescolares.

La pedagogía del tiempo libre trabaja con todas las edades, género y lugares. Se ocupan del desarrollo humano durante el tiempo de ocio o libre, ese tiempo que queda después del trabajo y del estudio (Fermoso, 1997).

Ahora bien, el tiempo libre y de ocio se asocia con las actividades recreativas. En este sentido, diferenciaremos cuatro tipos de recreación para delimitar la que nos interesa abordar en el ámbito de los Campamentos. Según Waichman (2008) ellas son:

a) La recreación y la animación se da como un simple entretenimiento en un tiempo de poca valía social y sin productividad. Es entonces, una actividad o grupo de actividades, que tienen lugar en un tiempo sin obligaciones laborales, que la persona selecciona y que le provocan placer.

b) Se enfoca sobre todo en el ámbito individual y puede trascender a lo grupal (por ejemplo, a la familia). Se caracteriza por la libertad de escoger las actividades a realizar durante el tiempo libre y sobre todo por la prioridad de hacer lo que a la persona le gusta. Por ejemplo, lectura y música.

c) El recreacionismo es el segundo concepto asociado a la recreación como una gran variedad actividades aleatorias que tienen como sentido el uso positivo y constructivo del tiempo libre. Tiene el único fin de divertirse y descansar de las tareas cotidianas. Nace de la Educación Física y como ejemplos están: visitar lugares públicos, hacer deporte y juegos virtuales.

d) La animación sociocultural nace en las postrimerías de la Segunda Guerra Mundial, inicialmente como metodología de educación para adultos y luego sería parte de la educación permanente o continua. Cuenta con conceptos asociados a la educación popular y participación social. Besnard (1991) define a la animación sociocultural como un método de organización del ocio en miles de prácticas culturales, estéticas, deportivas y sociales con diferentes fines políticos. Su énfasis está en el cambio sociocultural dirigido. Puede contar con diferentes formatos, por ejemplo: clubes, espectáculos culturales, fiestas populares y Campamentos.

e) La recreación educativa, a diferencia de la animación sociocultural, surge para las personas adultas, del ámbito de la educación no formal post-escolar o extraescolar y 
para ser complementaria de la escuela (una vez finalizada). Ahora bien, el autor plantea que la recreación educativa es hija de la animación sociocultural. Aquí la recreación es la educación en y del tiempo libre, estará pedagógicamente orientada y estructurada formalmente. Ejemplo: el Campamento.

Waichman (2008) señala que desde cada uno de los tipos de recreación citados anteriormente es posible organizar un programa de Campamento debido a sus principios de conceptuación de lo recreativo. Así las cosas, para la primera posición por ser una actividad saludable al aire libre; para la segunda por ser una tarea grupal movilizadora de formas culturales; para la tercera y cuarta posición son válidos los dos argumentos anteriores, pero incorporando la elaboración de objetivos educativos y planeamiento en el contexto de la formación permanente de los seres humanos y diferenciando los programas según la edad (este caso es perfecto para el ámbito de los Museos).

En este concepto de Campamento organizado, se combinan dos elementos esenciales: lo educativo y lo recreativo. Ambos en función de contribuir con la formación integral de sus participantes con sus fines, objetivos y procedimientos operativos y con la utilización de métodos y técnicas participativas.

Para complementar el planteamiento anterior nos referiremos a las características de la recreación educativa que señala Vigo (2005) y que son las siguientes:

a) Es voluntaria y libre (en tanto no es obligatoria por ley, sino de elección familiar o individual) no es compulsada.

b) Es una actitud de participación gozosa.

c) No es utilitaria en el sentido de esperar una retribución o ventaja material a cambio. Se hace por la sola satisfacción que produce. Esto por lo que sucede en el tiempo de no trabajo.

d) Es renovadora de las energías gastadas en el trabajo o en el estudio, porque produce distensión y descanso integral.

e) Es compensadora de las limitaciones y exigencias de la vida contemporánea, al posibilitar la expresión creadora del ser humano a través de las artes, las ciencias, los deportes y la naturaleza.

f) Es saludable, porque procura el perfeccionamiento y desarrollo del hombre. 
g) Es un sistema de vida, porque se constituye en la manera grata y positiva de utilizar el tiempo libre.

h) Es un derecho humano que debe ser válido para todos los períodos de la vida y para todos los niveles sociales.

i) Es parte del proceso educativo permanente por el que se persigue dar los medios para utilizar con sentido el tiempo libre.

j) Es algo que es organizado e incorpora a un grupo de personas.

k) Es una actividad de no trabajo y de libre selección.

Estas características, nos permiten construir un concepto de recreación educativa ligado al uso inteligente del tiempo libre, con otros y otras, al aire libre o en espacios cerrados y en función del disfrute organizado.

Referimos, entonces, al Campamento como un programa didáctico educativo no formal en el contexto de la recreación educativa, en tanto que, como afirma Parcerisa (2007), la educación no formal (o social) también necesita de procedimientos didácticos, aunque estos sean diferentes a los que utiliza la escuela, pero que, a la vez, complemente dicha formación.

En este sentido, Parcerisa (2007, pp. 45-46) señala que “...la didáctica es una disciplina científica que estudia los procesos educativos en ambientes organizados de relación y comunicación intencional, con el fin de orientar la mejora de su calidad".

En el caso de los Campamentos como educación no formal, se exige una didáctica apropiada a sus fundamentos, es decir, que propicie un tipo de comunicación entre las personas involucradas y que promueva el aprendizaje libre, flexible, creador y retador.

Así, las estrategias didácticas de Campamento deben estar acordes con la educación no formal y romper el esquema escolar, que necesita, según Castilla (2007, p. 77) las siguientes consideraciones:

- Ampliación del concepto de educación. Por ejemplo, incluir elementos recreativos con orientación pedagógica.

- Creciente estimación de valores educativos marginados por la escuela. Especialmente en el ámbito de la vida con otros y la autovaloración.

- Reconocimiento del valor formativo de la actividad lúdica. 
- Lucha pedagógica contra ocios nocivos. La idea de un campamento bien encauzado no es llegar a un desenfrenado "activismo divertido", sino utilizar elementos diversos para cumplir los objetivos pre establecidos.

Es importante señalar que no se registró investigación empírica de los procesos educativo-recreativos que se gestan en los programas de Campamento específicos. Los estudios realizados hasta el momento tienen el objetivo de evaluar los servicios del campamento. Por ejemplo: la alimentación, la inversión, las actividades favoritas y sugerencias.

Debido a la escasez de investigación en el ámbito de los Campamentos, resulta necesario promover estos estudios, para evidenciar el impacto educativo en las personas participantes y mejorar la pedagogía de la recreación educativa.

\subsection{El Campamento como programa didáctico de la educación No Formal}

En el Campamento organizado el sentido educacional es su esencia y el aspecto recreativo tendría una dimensión importante en la cuestión didáctica como recurso central. Esto se puede notar en los campamentos temáticos que se enfocan en contenidos técnicos, científicos o religiosos y que disponen de facilitadores capacitados. Sin embargo, las mismas instalaciones, como se verá más adelante, están diseñadas para realizar actividades deportivas y recreativas que suelen vincularse con los objetivos del programa didáctico.

Un ejemplo de lo anterior consiste en ofrecer un Campamento sobre dinosaurios y una sesión de juegos denominada "Dino-retos", donde a través de dinámicas lúdicas se destacan diferentes características de los dinosaurios. O, trabajar el libro Bíblico de Daniel en un campamento religioso y diseñar una sesión de juegos denominada "Las pruebas de Daniel" en donde se retoma, con estrategias didácticas, el contenido del libro.

En este sentido, Ruiz (2001) afirma que la más destacada característica del Campamento es la de ser una comunidad concebida, planificada y dirigida con un propósito educacional. En este sentido, el componente educacional es lo que lo distingue de experiencias de acampar en carpas y al aire libre como una experiencia familiar o de amistades.

Los Campamentos organizados se implementan desde la educación no formal, entendida como toda experiencia de enseñanza y aprendizaje previamente planificada con objetivos, contenidos, metodologías y actividades a realizarse en tiempo y espacios preseleccionados, con la participación de una población de beneficiarios que comparten ciertas características 
demográficas, socio-económicas e intereses temáticos. Así, por ejemplo, el Campamento del Museo de los Niños supone cinco meses de preparación.

Ahora bien, una modalidad de Campamento es el temático, donde con base en un tema se establecen unos objetivos y se planifican las principales actividades por desarrollar. Algunos ejemplos de estos programas serían los siguientes:

a) Un Campamento deportivo como el caso de Camp Bounedale en Massachusetts (http://www.campbournedale.com/default.asp)

b) Un Campamento de computación ofrecido en varias universidades estadounidenses denominado iD Teach Camps (http://www.internaldrive.com/)

c) Un Campamento de Música como es el Rock and Roll Camp en Atlanta (http://www.mysummercamps.com/camps/Detailed/Camp Jam - Atlanta L29022.html)

d) Un Campamento de ajedrez por ejemplo el USA Chess Camp (http://www.chesscamp.com/? $\mathrm{p}=$ services camps)

Se puede notar, entonces, que cualquier tema puede ser utilizado para planificar e implementar un programa de Campamento educativo, siempre y cuando se enfatice en los principios de la administración y educación no formal indicados en este artículo.

Otro componente esencial de la educación no formal, y que es un asunto vital en un Campamento, es el equipo de facilitadores, guías o líderes capacitados para la promoción del aprendizaje activo y vivencial. Al respecto, Lorenzo (2007) indica que el liderazgo en las organizaciones educativas no formales tiene la función de dinamizar a un grupo o a una organización para generar su propio crecimiento y logro del cumplimiento de su misión o proyecto compartido.

Para que los líderes de los Campamentos puedan lograr este proceso dinamizador del programa necesitan una identificación y motivación con los fines y los objetivos del Campamento, condición fundamental. En otras palabras, además de su preparación técnica específica en un área temática determinada, el liderazgo debe proyectar el gusto y el disfrute personal por aquellos temas y valores que el Campamento desea promover entre los campistas; esto de manera transversal durante toda la ejecución del programa.

De igual manera, debe haber coherencia entre los temas y las actividades de apoyo logístico. Así, por ejemplo, si se trabaja el tema "Anatomía humana" y la importancia de la sana alimentación, obviamente el programa debe ofrecer un menú consecuente con lo enseñado. $\mathrm{Si}$ 
se abordan los valores de género, se debe promover entre el liderazgo y demás participantes el respeto mutuo en la forma de humor durante los espacios libres del programa y demás comportamientos afines con los valores.

Para lograr lo anterior es importante la capacitación de los facilitadores para mantener un equipo de liderazgo base estable, que contribuya a fortalecer y mantener aquellos valores y componentes señalados como importantes en la cultura del campamento.

En el caso de la autora y el autor que han participado en la organización de campamentos en varias instituciones, en todos los casos se realizó una capacitación para todas las personas que trabajaron como facilitadores o líderes de grupo. Estas capacitaciones varían en duración, de 4 horas a 32 horas, por lo general depende de la cantidad de personal nuevo y sin experiencia.

La exploración y la promoción del aprendizaje orientado por objetivos en un Campamento son componentes centrales, así aprender haciendo, aprender jugando, aprender por descubrimiento con base en normas y valores para la convivencia se llegan a entrelazar con la dimensión recreativa. Ese, en nuestro criterio, es el "secreto" de su diseño y el éxito que permite la continuidad de este programa en el transcurso del tiempo.

En este punto, interesa plantear una propuesta que desglose y describa los ejes teóricometodológicos que deben caracterizar un programa de Campamento desde la educación no formal y anotarlos como componentes y elementos esenciales para su diseño.

Los ejes teórico-metodológicos que la autora y el autor han utilizado en el diseño e implementación de Campamentos y que se platean como esenciales, son descritos en los siguientes títulos.

\section{Aprender haciendo}

El concepto de Educación Activa, aplicado al valor educacional del Campamento organizado, está estrechamente ligado a lo que planteó Dewey (1938) sobre la educación como el medio para adquirir conocimientos y aplicarlos para una vida mejor. Este autor plantea que la clasificación y el ordenamiento de experiencias afectan favorable e inteligentemente la conducta del educando, pues éste es el principio de aprender haciendo.

Paris (2008) argumenta que el proceso educativo en un Campamento, a diferencia de la educación formal tradicional, consiste en guiar a las personas participantes en su aprendizaje 
por medio de la participación activa. Así, la educación es vida, en contraposición a la idea de que es una preparación para la vida.

El concepto de "aprender haciendo" será el eje de la filosofía educativa de los Campamentos organizados, este es un eje transversal de toda la planificación y accionar del programa didáctico. Todo tema tratado cuenta con actividades participativas para poner en práctica los conceptos básicos.

El movimiento Scout incorpora plenamente este concepto, el aprendizaje por acción refleja un enfoque activo que ayuda a los jóvenes a desarrollarse por medio de oportunidades, de experiencias directas y concretas, en oposición a escuchar o ver demostraciones pasivas. Se aplica a la forma en que los jóvenes desarrollan conocimientos, destrezas y actitudes para progresar hacia los objetivos educativos planteados.

El aprendizaje mediante la acción no está, por lo tanto, limitado a "hacer", en el sentido de aprender destrezas prácticas y manuales, sino implica también lo que en inglés se denomina "minds on", o sea, es posible y necesario participar con la mente. Por ejemplo, los jóvenes aprenden el significado de la responsabilidad, asumiendo responsabilidades. (http://www.gsvd.d/talleres-libros/documentos\%20/sistema-educativo.pdf recopilado el 26 de febrero 2009).

Algunos recursos didácticos utilizados en los Campamentos que refuerzan esta idea son los acertijos (más adelante los detallaremos) y los estudios de Biblia participativos mediante guías de discusión.

\section{Aprender jugando}

El proceso de aprender está presente durante toda la vida del ser humano, en diferentes espacios y actividades socioculturales. Tradicionalmente, se entiende desde la educación formal que el único espacio de aprendizaje es el aula escolar de primaria para el caso de la población infantil, el colegio para la juvenil de secundaria y la universidad para jóvenes-adultos.

Sin embargo, la concepción espacial y modalidad educacional no formal e informal han ampliado el concepto educativo, en el sentido de que sabemos que construimos conocimiento mediado por la tecnología o los objetos, la actividad (incluyendo el juego) y otras formas de interacción social durante toda la vida. Se aprende en todos los espacios socioculturales, con los medios de comunicación colectiva, mediante las Tecnologías de la Información y de la 
Comunicación (TIC), en los múltiples formatos que la Internet posibilita, en actividades culturales, artísticas y lúdicas.

Aprender jugando implica la adquisición de conocimientos, habilidades, destrezas, actitudes y valores mediante diversos juegos que utilizamos con unos objetivos pedagógicos concretos. Así, los juegos de mesa (por ejemplo, Cranium y Pictionary) pueden ser usados para trabajar temas como la comunicación. Los juegos tradicionales para abordar temas de competencia y género. Para trabajar actitudes de colaboración y valores para la convivencia social, juegos tales como rallies de retos y de acertijos. También se pueden utilizar juegos electrónicos para abordar temas tales como apreciación de arte o economía.

El juego es el recurso central para diseñar las actividades que integran el programa de un Campamento; de la forma más divertida y entretenida se construyen nuevos conocimientos, se aprende a respetar las reglas, a ser tolerante ante la adversidad, a trabajar colaborativamente para el logro de objetivos, a competir sanamente, se fortalece el auto conocimiento (limitaciones y fortalezas); y cualquier tema contenido en los objetivos predeterminados. Los Campamentos tienen en los juegos educativos y actividades recreativas su fundamento para la integración grupal y el desarrollo de la personalidad, además de que le da el sentido recreacional requerido ya que no hay que perder de vista que se ejecuta en tiempo de vacaciones.

En suma, jugar es otra forma de aprender haciendo y de aprender por descubrimiento en los términos que exponemos a continuación.

\section{Aprender por descubrimiento}

El protagonismo de las personas participantes en el Campamento es un eje orientador del diseño de las acciones educativas y actividades recreativas implementadas en los diversos espacios y horarios programados.

El principio del aprendizaje por descubrimiento es opuesto al aprendizaje por recepción; en el primer caso las personas tienen el protagonismo en el proceso educativo; en el segundo caso, tal y como lo plantea Madruga (2003), en el aprendizaje por recepción la persona recibe los contenidos que debe aprender en su forma final, acabada; no necesita realizar ningún descubrimiento más allá de la comprensión y de la asimilación de los mismos de manera que sea capaz de reproducirlos cuando le sea necesario. Contrario a lo anterior, el aprendizaje por descubrimiento implica una tarea distinta; el conocimiento no se da en su forma acabada, sino 
que debe ser descubierto. Desde este planteamiento pedagógico resulta claro comprender por qué debemos incluir en las actividades del Campamento educativo retos y desafíos que casualmente impliquen acciones de las personas encaminadas a descubrir por ellas mismas las respuestas, plantearse preguntas, cuestionarse situaciones, involucrarse en la búsqueda de información y descubrir la mejor solución posible a los problemas de acuerdo con las exigencias de las actividades.

El experimento como recurso didáctico (Rodríguez y Vargas, 2009) es un insumo muy valioso para el descubrimiento, las competencias y los debates permiten elaborar cierres de temáticas polémicas importantes para la edad en donde al interior del grupo se construyen conceptos y reafirman valores.

\section{La colaboración y la cooperación}

El Campamento es un espacio social diseñado culturalmente para convivir con otros pares en tiempo vacacional y con unos objetivos compartidos entre quienes organizan el programa y las personas participantes.

La actividad social supone dos componentes centrales: cooperar y competir. Ambos aspectos, en el contexto del Campamento educativo, están presentes de manera transversal en la programación y en la ejecución de las diversas actividades con el propósito de fomentar habilidades sociales relacionadas con los juegos cooperativos, el trabajo en equipo, las dinámicas de grupo, y la promoción de ambientes competitivos en sintonía con el cumplimiento de la reglamentación respectiva. Vigo (2005, pp. 4-5) afirma que:

(...) debe señalarse el hecho de la vida en grupos. Este proceso socio-educativo es el contenido principal del Campamento organizado. Exceptuando que el comportamiento del ser humano está motivado en mucho por el deseo de ser aceptado y lograr una posición entre sus iguales, y que, como respuesta, es en el grupo congeniante de sus iguales donde el individuo encuentra la plena expresión de sus necesidades personales, es que se puede comprender la magnitud de los recursos potenciales del Campamento para la socialización del acampante. Digamos, resumiendo hasta aquí, que esta comunidad de propósito educacional (...) constituye la característica central del Campamento organizado. 
Al respecto, Onrubia (2009) señala la necesidad de estructurar el proceso de cooperación y colaboración para aprender cómo participar en este tipo de situaciones y para fomentar el aprendizaje. Actividades colaborativas incentivan la toma de decisiones conjuntas. Para esto se aumenta la frecuencia de conflictos socio cognitivos mediante retos, acertijos u otras técnicas similares que fomenten la elaboración de explicaciones y la comprensión mutua.

En síntesis, estas orientaciones teórico metodológicas en el contexto de la educación no formal, permiten hilvanar el tejido conceptual del Campamento Educativo para fomentar procesos de enseñanza-aprendizaje orientados por objetivos centrados en la interacción grupal. Podemos afirmar que el juego, el aprender haciendo y aprender por descubrimiento en actividades de competencia y colaboración son los recursos fundamentales de la planificación y de la ejecución de las actividades de un Campamento.

\subsection{El liderazgo y la formación de facilitadores}

Según se indicó anteriormente el espíritu del Campamento es promovido por las personas facilitadoras que son los líderes y las líderes de grupos pequeños. Gran parte de ese ambiente colectivo de un Campamento lo da el carisma de estas personas, su identificación con el tema, con la cultura de Campamento y su capacidad por compartir todo lo anterior con el grupo bajo su responsabilidad. Hemos visto en nuestra experiencia de ejecución de campamentos, en el Museo de los Niños, a varias personas facilitadoras con carisma y habilidades de liderazgo hacer de un atraso en el programa el rato más divertido y consecuente con el tema del campamento, al punto que los niños y las niñas no notan lo sucedido.

Tanto Incarbone y Guinguis (2006) como Vigo (2005) consideran el liderazgo como la característica que identifica la calidad del personal en cuyas manos se ha de confiar la dirección del Campamento, representa algo muy delicado e importante, pues considerando al Campamento como un proceso educativo, es obvio que éste debe ser dirigido por personas idóneas.

Las competencias esenciales que deben tener los líderes de los Campamentos están definidas en función de las actividades y rasgos específicos de cada Campamento en concordancia con sus diferentes énfasis: deportivos, recreativos, artísticos, científicos, culturales, espirituales, entre otros.

La personas líderes, guías y facilitadoras que participan en la organización y realización de los Campamentos deben ser competentes en el manejo de técnicas participativas grupales, 
creatividad para improvisar de manera oportuna y pertinente, y sobre todo pasión y afinidad con el tema central del programa. Su formación ética y moral debe ser apropiada para implementar de manera transversal los valores del Campamento entre el grupo.

La formación de las personas líderes de los Campamentos debe enfocarse en las metodologías participativas por implementar, es necesario que cuenten con cualidades personales para trabajar en los Campamentos, tales como: habilidades sociales para el trato con campistas y para trabajar en grupo, disponibilidad de horario, posibilidad de rendir cuentas, capacidad de lograr metas en ambientes de trabajo con ritmos acelerados y bajo presión. Iniciativa para sugerir actividades coherentes con la vida y el tema del Campamento, así como creatividad para plantear soluciones viables ante los problemas imprevistos del accionar de los Campamentos.

\section{Principales aspectos de la administración de la educación no formal aplicados a un programa de Campamento}

Para Vigo (2005) el proceso administrativo del Campamento está compuesto por cinco pasos fundamentales que están muy acorde con nuestra experiencia. A continuación se detallan:

a) Formulación de propósitos y objetivos. Son la guía para la acción. Son parte del planeamiento.

b) Programación. Es la determinación de los recursos, tipo y funciones del personal, el tiempo, duración y tipo de actividades, los espacios físicos a utilizar, los requerimientos de equipamiento, seguridad, salud, finanzas. La atención a las necesidades sociales y la posibilidad de flexibilizar los programado para adaptarse a las modificaciones e imprevistos sin mayor dificultad.

c) Organización. Consiste en crear y mantener una estructura que facilite su funcionamiento, ejecutar los procedimientos de acuerdo con lo establecido en el planeamiento, se coordina la realización de las actividades, se recluta el personal necesario, se formulan pautas de trabajo, procedimientos y rutinas, se establece la responsabilidad de que se opere en forma económica y eficiente, alerta para detectar y corregir situaciones de riesgo.

d) Dirección ejecutiva. Es la etapa de supervisión para cerciorarse de que se cumplan las condiciones necesarias para alcanzar las metas propuestas, le corresponde a esta 
instancia trasladar a la acción los objetivos, los planes y los métodos que han sido enunciados. En esta etapa estaría la ejecución del Campamento por parte del equipo de trabajo.

e) Evaluación. Establece el grado de cumplimiento de los objetivos y metas propuestos, los aspectos a mejorar, sirve para promover nuevos planteamientos, nueva organización y ejecución, y reafirma los logros y metas cumplidas.

Es importante resaltar que en los Campamentos organizados los objetivos o propósitos son el eje central de todo el proceso educativo y administrativo, pues permiten contar con un norte a seguir, o sea, son los lineamientos orientadores de todos los procesos técnicos administrativos. Vigo (2005) sugiere los siguientes propósitos del Campamento:

a) Ofrecer una experiencia educativa de vida rústica (o diferente al estilo de vida de las personas participantes) en un contexto de familiaridad en términos de relaciones interpersonales, pero con el impacto de las actividades novedosas y de aventura en espacios no cotidianos.

b) Mejorar la salud mediante un sistema de vida adecuado, alimentación sana y equilibrada, descanso suficiente y actividad física de intensidad controlada, conduciendo al campista a la adquisición de hábitos de vida saludable. Esto se refiere a los campamentos de montaña específicamente donde este tema es un eje transversal (por ejemplo, el Campamento Scout y el de Roblealto).

c) Propiciar nuevas posibilidades para el uso constructivo del tiempo libre, dando a cada campista la oportunidad para aprender habilidades físicas, sociales, manuales, entre otras que le permitan un desarrollo humano integral.

d) Contribución con el desarrollo de la personalidad, proporcionando un ambiente social donde cada campista pueda encontrar sus propios medios o vías de expresión, fortalecer sanas costumbres sociales que le permitan una relación satisfactoria con los demás, y lograr emancipación emocional de padres, madres y encargados de crianza.

e) Estimular el pensamiento creador mediante formas de educación activa, provistas por el medio natural de la vida de Campamento, y por los grupos y núcleos que conforman la estructura de su organización.

Estos propósitos de Campamento deben contextualizarse a cada institución, adaptarse a los temas que se desarrollen y a las características de los participantes. 


\section{Campamentos educativos en Costa Rica}

En Costa Rica, desde la primera mitad del siglo pasado, se realizan Campamentos. A continuación, en el Cuadro No. 3 se presentan algunos de los principales Campamentos pioneros que contaron con instalaciones destinadas para esta actividad en Costa Rica, según nombre, año de fundación, ubicación y dirección web en caso de que deseen observar la infraestructura:

\section{Cuadro No. 3.}

Principales instalaciones destinadas para Campamentos en Costa Rica, según nombre, año de fundación, ubicación y dirección web.

\begin{tabular}{|l|c|l|l|}
\hline \multicolumn{1}{|c|}{ Nombre } & $\begin{array}{c}\text { Año de } \\
\text { fundación }\end{array}$ & \multicolumn{1}{|c|}{ Ubicación } & \multicolumn{1}{c|}{ Dirección web } \\
\hline $\begin{array}{l}\text { Campamento Roblealto, } \\
\text { Asociación Roblealto }\end{array}$ & 1948 & $\begin{array}{l}\text { Barva de } \\
\text { Heredia. }\end{array}$ & www.roblealto.com \\
\hline Campamento Bautista & 1973 & $\begin{array}{l}\text { San Rafael de } \\
\text { Alajuela. }\end{array}$ & www.campamentobautista.org \\
\hline $\begin{array}{l}\text { Campamento } \\
\text { La Garita }\end{array}$ & 1975 & $\begin{array}{l}\text { La Garita de } \\
\text { Alajuela. }\end{array}$ & www.campamentocristianolagarita.co \\
\hline $\begin{array}{l}\text { Campamento } \\
\text { La Cumbre } \\
\text { (antes Savegre). }\end{array}$ & 1975 & $\begin{array}{l}\text { San Gerardo de } \\
\text { Dota. }\end{array}$ & www.lacumbredota.org \\
\hline $\begin{array}{l}\text { Campamento Campo } \\
\text { Escuela Iztarú, } \\
\text { Movimiento Mundial } \\
\text { Scout. }\end{array}$ & 1978 & $\begin{array}{l}\text { Tres Ríos de } \\
\text { Cartago. }\end{array}$ & www.siemprelistos.com \\
\hline $\begin{array}{l}\text { Campamento } \\
\text { Vida Joven. }\end{array}$ & 1983 & $\begin{array}{l}\text { Esterillos de } \\
\text { Puntarenas. }\end{array}$ & www.vidajovencr.org \\
\hline $\begin{array}{l}\text { Campamento Oikoumene } \\
\text { (YMCA) }\end{array}$ & 1990 & $\begin{array}{l}\text { Ochomogo de } \\
\text { Cartago. }\end{array}$ & www.campamentooikoumene.org \\
\hline $\begin{array}{l}\text { La Montaña Christian } \\
\text { Camps }\end{array}$ & 1998 & $\begin{array}{l}\text { Bajo Los } \\
\text { Rodríguez, San } \\
\text { Ramón de } \\
\text { Alajuela. }\end{array}$ & www.lamontana.net \\
\hline
\end{tabular}

Fuente: Elaboración propia con base en experiencia de la autora y el autor.

Según se puede apreciar en el Cuadro No. 3 entre los pioneros costarricenses del movimiento campista con instalaciones específicas está el Campamento Roblealto de la Asociación Roblealto, quienes han recibido sus capacitaciones de la Asociación de 
Campamentos Cristianos Internacionales (www.ccial.org) organización que cuenta con una serie de publicaciones sobre técnicas educativas para campamentos y promueven la cultura campista en el ámbito internacional.

Las instalaciones diseñadas y construidas para hacer campamentos, por lo general, contienen: cabañas (para alojar a campistas y a líderes), salones multiusos (se pueden usar como comedor), cocina, piscina o lago, tobogán de agua, piscina de barro, canchas deportivas, instalaciones para deportes extremos, salón de patines, área de fogatas, senderos y construcciones especiales para implementar acertijos ${ }^{3}$

El Movimiento Scout de Costa Rica es el pionero en realizar Campamentos educativos, iniciándose en 1915, pero sin la infraestructura con la que cuentan desde 1978. Además, es importante destacar que no se registraron investigaciones sobre historia del Campamento en el ámbito nacional.

\section{Museos y Campamentos}

No se registraron muchas publicaciones sobre la historia del vínculo de los Museos y los Campamentos. Si bien el inicio de los departamentos educativos en museos se da con el impulso de Molly Harrison y se implementó como un fenómeno sociocultural masivo en la década de los años 70 (Pastor, 2004) es importante señalar que los Campamentos y otras expresiones educativas en Museos se vienen construyendo desde finales del siglo XIX. Hailey (2009) refiere los campamentos de museos como un tipo más entre los actuales.

Es obvio que los Museos no diseñan el "Campamento" como estrategia didáctica, se lo apropian y le dan sentido en el contexto de su colección y sus exhibiciones, utilizando sus temas de interés y haciendo uso de las estrategias pedagógicas mencionadas en el apartado anterior y también implementadas en Campamentos de otras latitudes.

En el caso de los reconocidos teóricos de la educación de museos estadounidenses Falk y Dierking, ellos incursionan en Campamentos y luego en Museos. Esos autores afirman que antes de ingresar al ámbito museal, tienen sus primeros contactos con la educación no formal en los Campamentos de verano del movimiento "The Girl Scout". Señalan que esa experiencia les da un impacto en su proceso de comprensión del fenómeno del aprendizaje informal que en

3 Son juegos cooperativos que cuentan con un marco mágico o historia que sirve de contexto para desarrollar la actividad. Al finalizar la actividad se reflexiona sobre lo sucedido mediante la técnica DIA (Descripción de los sucedido, interpretación y aplicación a la vida personal y grupal). 
el transcurso del tiempo ha sido plasmado en muchos textos, investigaciones y producción científica (Katz, 2001)

Ahora bien, según lo consultado el registro más antiguo de un Campamento en Museos lo ofrece Danilov (2005) cuando cita que en el año 1925, la señora Helen Mc Graw, en el Mc Graw Memorial Park, dirigía ahí Campamentos de verano.

En el caso del Museo de los Niños, la autora y el autor antes de su experiencia en el Campamento de este Museo, laboraron como líderes y facilitadores en el movimiento Scout y otros Campamentos juveniles. Dicha experiencia proveyó el insumo básico para el desarrollo y consolidación del actual programa de dicho Museo.

Uno de los aspectos importantes de los Campamentos en Museos es que resulta una forma de obtención de recursos económicos para financiar las necesidades operativas y logísticas museales. Este programa, además, está acorde con los fines educativos de la institución. Falk y Dierking (2000) ya han señalado que en la sociedad del consumo, diferentes formas de aprendizaje es un servicio que se puede ofrecer y para los museos es una fuente de recursos viable, aceptable y armoniosa con sus objetivos educativos.

\subsection{Los Campamentos Urbanos: espacios y actividades}

La diferencia central entre el Campamento Urbano o Day Camp y el Campamento clásico reside en que, en el primer caso, las personas campistas por lo general no se quedan a dormir ${ }^{4}$ y suele implementarse en la ciudad e inclusive puede tratarse de un programa de sólo un día. Han sido muy promovidos por museos urbanos.

Hailey (2009) refiere que algunos campamentos urbanos en los Estados Unidos se han propuesto establecer el vínculo entre la vida de la cuidad y la naturaleza, mientras que otros se han enfocado en el arte. Consideramos que también el tema de la ciencia en los Museos de los Niños y de las Ciencias es otro de los temas abordados. Por ejemplo, el caso de los Museos de la Caixa que en varios lugares de España ofrecen este tipo de campamentos (Rodríguez, 2005)

La definición de las actividades y los espacios requeridos para un Campamento dependen del tema y de los objetivos de los talleres. El simple hecho de que sea en la ciudad ya es una limitante para unas actividades, pero no para otras. Así, el espacio tiene un papel importante en tanto que puede limitar o potenciar el tipo de actividades y temas posibles.

4 Hailey (2009) señala una excepción para Estados Unidos.

Volumen 11, Número 1, Año 2011, ISSN 1409-4703 
Si interesa que los participantes tengan contacto con las ciencias naturales y sus diferentes campos, la educación ambiental se implementa utilizando las facilidades de centros de investigación, laboratorios, planetarios, museos, viveros, invernaderos, fincas experimentales, zonas verdes, parques, edificios patrimoniales, jardines botánicos, entre otros similares.

Otelo e Iglesias (1991) indican que en el caso de España, los Campamentos Urbanos se inician e Cádiz con ocasión del Año Internacional de la Juventud en agosto de 1985. El segundo programa de esta modalidad se efectuó el siguiente año en el Año Internacional de la Paz. De ahí la experiencia se extiende a todo el país. No se registraron referencias sobre el origen de esta modalidad en otros países.

En cuanto a la metodología eminentemente participativa, para el caso de la ciencias naturales se suele utilizar el experimento científico como recurso didáctico (Rodríguez y Vargas. 2009) y las demostraciones de fenómenos sorprendentes para promover un acercamiento amigable y divertido. Estas experiencias, sin exámenes y en un ambiente de juego y exploración, generan aprendizajes significativos 0 , al menos, la motivación de las personas campistas para profundizar en el mundo de la física, biología, química, astronomía, matemáticas, geometría, entomología, geología, entre otras.

Los Campamentos Urbanos incluyen actividades al aire libre; juegos tradicionales, juegos y dinámicas de grupos, retos, desafíos, actividades deportivas y recreativas. Éstas han sido previamente planeadas con énfasis en la interacción social, la puesta en práctica de los valores para la convivencia y el cumplimiento de las reglas que los mismos juegos señalan.

\subsection{La experiencia del Museo de los Niños de Costa Rica: El Campamento Urbano "Vacaciones Felices"}

En este apartado se expone sobre el Campamento Urbano del Museo de los Niños de Costa Rica, denominado Vacaciones Felices; su origen, sus objetivos y su organización.

El Campamento de Vacaciones Felices nace en enero de 1999 como una forma de explotar las salas del Museo de los Niños de manera más vivencial. Los objetivos para el programa, que la autora y el autor establecieron en el plan de trabajo institucional, son:

a) Ofrecer un programa vacacional en un formato de Campamento Urbano para el aprovechamiento inteligente del tiempo libre. 
b) Satisfacer la necesidad de los padres y madres de una "guardería" para sus hijos e hijas mediante un Campamento educativo.

c) Promover el gusto por la ciencia, el arte y las habilidades sociales para la vida con otros.

d) Generar espacios educativos donde predomine lo lúdico y el disfrute con otros.

En la actualidad este programa supone la realización de 400 talleres por año totalmente novedosos, respecto a los ofrecidos en el año anterior. Los grupos de edad han cambiado en el tiempo. Actualmente, se trabaja con cuatro grupos: niños y niñas de 3 años y 6 meses a 4 años y 11 meses; de 5 a 6 años; de 7 a 8 años; de 9 a 10 años y de 11 a 13 años. Cada grupo cuenta con un programa de actividades diferente y adecuado a sus intereses y habilidades, pero con temas similares.

El programa está organizado por talleres, cada uno de los cuales cuenta con actividades que utilizan las salas museográficas del Museo de los Niños, aunque no es exclusivo.

\section{Planificación y organización}

Vacaciones Felices es uno de los programas del Museo de los Niños que requiere de una mayor coordinación inter departamental y del tiempo, puesto que termina una temporada e inmediatamente inicia la planificación de la siguiente. Para su ejecución, se ha contado con una coordinación general, un asistente de Campamento, un asistente de logística, 13 guías de grupo (cada grupo de niños oscila entre 8 a 10 niños y niñas, según la edad), 3 asistentes por grupo de edad, siete facilitadores y apoyo administrativo de 3 personas. Adicionalmente, se cuenta con el apoyo permanente de 10 a 12 agentes de seguridad y de primeros auxilios. Por su parte, están los departamentos de Financiero, Adquisiciones y Servicios Generales que apoyan en tareas administrativas.

En el siguiente Cuadro No. 4 se describe la planificación y la organización del Campamento según los niveles educativo-recreativos, de requerimiento de personal, y de procedimientos administrativas, y las tareas que cada uno de los niveles supone: 


\section{Cuadro No. 4. \\ Niveles y tareas en el proceso de planificación y organización del Campamento Urbano "Vacaciones Felices" del Museo de los Niños.}

\begin{tabular}{|c|c|}
\hline Nivel & Tarea \\
\hline \multirow{6}{*}{ Educativo-recreativo } & Planificación de temas. \\
\hline & $\begin{array}{l}\text { Planificación didáctica del programa general por edades. } \\
\text { Cronograma. }\end{array}$ \\
\hline & Diseño de talleres por edad. \\
\hline & Planificación de recursos didácticos por edad. \\
\hline & Revisión de propuestas educativas. \\
\hline & $\begin{array}{l}\text { Diseño de actividades para situaciones de emergencia } \\
\text { (ausencia de un facilitador o facilitadora). }\end{array}$ \\
\hline \multirow{3}{*}{ De personal } & Selección de líderes de grupos por edad. \\
\hline & Selección de asistentes. \\
\hline & $\begin{array}{l}\text { Delimitación de temas y talleres según formación académica } \\
\text { de facilitadores y facilitadoras. }\end{array}$ \\
\hline \multirow{8}{*}{ Tareas administrativas } & Proceso de matrícula. \\
\hline & Preparativos de listas según la edad. \\
\hline & Solicitudes de bienes y servicios con suficiente antelación. \\
\hline & $\begin{array}{l}\text { Coordinación del apoyo de otros departamentos (limpieza, } \\
\text { seguridad, recursos necesarios, mercadeo, etc.). }\end{array}$ \\
\hline & $\begin{array}{l}\text { Coordinación de uso de espacios para el desarrollo del } \\
\text { Campamento. }\end{array}$ \\
\hline & $\begin{array}{l}\text { Coordinación de uso equipo para talleres en el tiempo y el } \\
\text { espacio. }\end{array}$ \\
\hline & $\begin{array}{l}\text { División del trabajo para divulgar el Campamento. Reglamento } \\
\text { interno del trabajo según puestos. }\end{array}$ \\
\hline & Consecución de donaciones. \\
\hline
\end{tabular}

Fuente: Elaboración propia.

Según lo anotado en el Cuadro anterior, realizar un Campamento necesita mucha planificación y organización para que todos los recursos puedan estar a tiempo y sea adecuado y seguro para los niños y las niñas, principales beneficiarios de este programa educativo.

El Campamento Vacaciones Felices se inserta en la modalidad de Campamentos Urbanos, que según lo indicado en el punto anterior son programas que se desarrollan en medios urbanos, como bien lo aclara su nombre, y donde las personas participantes no se quedan a dormir como sucede en los Campamentos tradicionales. Lo anterior explica el por qué 
de un Campamento Urbano en el Museo de los Niños, aunado al hecho de que no se cuenta con infraestructura adecuada para el hospedaje de los niños y las niñas.

El Centro Costarricense de Ciencia y Cultura, sede del Museo de los Niños, cuenta con cuatro proyectos ubicados en cada uno de los cuatro pabellones: el Auditorio Nacional, el Complejo Juvenil del Conocimiento, la Galería Nacional y el propio Museo de los Niños. Este complejo cultural se ubica en la antigua Penitenciaría Central de San José y cuenta con condiciones arquitectónicas para velar por la seguridad de los niños y las niñas, así como con espacios verdes y recursos didácticos sumamente diversos y atractivos acordes con la naturaleza de los proyectos que conforman el Campamento.

El hecho de recibir y devolver a más de 100 niños y niñas por día a sus respectivas personas encargadas, necesita de un sistema de seguridad bastante elaborado para la protección de las personas menores de edad en medio de un espacio público. Asimismo, este sistema de seguridad contempla el cuido durante todo el día y los mecanismos de atención de situaciones de emergencia.

Además, una nueva modalidad para la planificación del Campamento es la de utilizar un eje temático que sirva de norte. El "tema" puede ser de contenido o de recurso metodológico. Por ejemplo, en los recursos didácticos hemos utilizado al teatro y en los contenidos propiamente el tema de los Dinosaurios.

Este programa de Campamento exige una organización del trabajo de parte del personal involucrado en la delimitación del uso de los espacios, en la selección de los temas de las actividades, en el uso de recursos tecnológicos, en la compra y preparación de materiales y en la programación de las actividades educativas.

De igual manera, supone la preparación de actividades para situaciones de emergencia, por ejemplo, que algún facilitador o facilitadora no se pueda presentar a realizar uno de los talleres que conforman el programa de Campamento.

Para diseñar un programa de un Campamento se necesita invertir más tiempo de lo que implica la ejecución del programa. La tarea de ubicar actividades participativas y cooperativas o de competencia que sean suficientemente atractivas para captar la atención de los niños y las niñas según su edad y sin importar sus diferentes gustos e intereses resulta muy compleja. Se logra gracias a la experiencia de los facilitadores y administradores del componente educativo del programa, que permita el equilibrio perfecto de "aprender jugando". 
Entendemos, entonces, que la recreación es un recurso más en el proceso educativo y que el juego pedagógicamente orientado se constituye en una estrategia central para hacer del aprendizaje pedagógicamente orientado una aventura socio-cognitiva.

Un programa de Campamento ofrece las condiciones para diseñar una experiencia pedagógica donde el jugar y aprender van de la mano, lo cual es propio del tiempo vacacional.

\section{Evaluación del proceso}

El tipo de evaluación que Vacaciones Felices utiliza es de proceso. Según Arco y Fernández (2007, p. 675): "La evaluación de proceso es aquella dirigida a analizar y comprobar la secuencia de elementos y actuaciones llevadas a cabo durante el diseño, planificación y aplicación del programa y compararlos con lo inicialmente previsto". Este tipo de evaluación supone reuniones diarias, durante su ejecución con la coordinación del Campamento, quienes asisten, los facilitadores y las facilitadoras de talleres y las personas que lideran los equipos, el personal de Seguridad y de Servicios Generales. En estas reuniones se evalúa la ejecución de la programación, la seguridad de los niños y las niñas, el trabajo de equipo, los tiempos de alimentación y se reciben sugerencias para el mejoramiento continuo y ajustes correspondientes a realizar en el siguiente día de programación del campamento.

Todos los cambios que se necesitan para el buen funcionamiento del programa se ejecutan en la medida de lo posible inmediatamente. En este sentido, cada facilitador y facilitadora recibe una retroalimentación de sus talleres de parte de las personas que lideran grupos, con el propósito de mejorar el taller que corresponda para la siguiente semana.

Un aspecto que se toma en consideración para determinar el éxito del programa es la cantidad de personas matriculadas, las inconformidades señaladas en los reclamos y recomendaciones de los padres y las madres de familia, a quienes se les contacta a la hora de recibir y entregar a los niños y a las niñas cada día.

También se han utilizado instrumentos (cuestionarios y guías de entrevista) para recoger opiniones que permitan calificar el programa de parte de los padres y las madres. Uno de los puntos que se ha mejorado notablemente es la alimentación (menú) que fue una queja permanente. Se logró resolver satisfactoriamente y de manera práctica según las demandas y posibilidades del programa. 
Uno de los éxitos de este programa es la capacidad de cambio en el proceso de ejecución, para poder mejorar en el momento que se presenta un reto. Esto se logra mediante la evaluación de proceso al finalizar la jornada diaria.

Al respecto, Luján (2010, p. 116) anota:

Es importante destacar que existen diversas metodologías para realizar las evaluaciones de los procesos educativos. Interesa indicar su funcionalidad en la comparación entre lo planeado y lo realizado, lo que se ejecuta durante la marcha de la acción educativa para encontrar las discrepancias que se producen en ese momento, a fin de tomar las decisiones para ajustar la acción educativa y mejorar el desempeño del (programa) y el éxito de la población participante.

Es importante resaltar que el ajuste en una evaluación de proceso es inmediato y en función de facilitar el logro de los objetivos y las metas definidos previamente o la incorporación de nuevos elementos y procesos en beneficio del mejoramiento de la calidad de la experiencia educativa.

\section{Conclusiones}

\subsection{El Campamento como programa didáctico elementos teórico-metodológicos para su implementación en los Museos}

El Campamento ha sido un recurso educativo-cultural importante en la vida de miles de personas para el uso inteligente de su tiempo libre y para la educación recreativa de la niñez y juventud.

En el transcurso del tiempo ha pasado de ser una actividad meramente recreativa, a ser un programa didáctico con una dimensión educativa no formal, orientada pedagógicamente hacia la promoción de habilidades sociales y del aprendizaje vivencial de temas diversos.

De la revisión bibliográfica realizada en diferentes contextos y de la experiencia del autor y la autora, se puede afirmar que los programas de Campamento actuales se conciben con esa combinación equilibrada de lo recreativo y lo educativo. Así, aprender haciendo, mediante el juego, con otros y otras, tiene un sentido vinculado al uso inteligente del tiempo libre y a la promoción de habilidades sociales.

La administración de la educación no formal permite concebir al programa de Campamento en sus diversas fases de planificación, organización, ejecución y evaluación. Este 
enfoque permite entender que el Campamento es un espacio desde donde se posibilita e impulsa un diseño didáctico que facilita el aprendizaje vivencial y lúdico, dando especial importancia a la orientación pedagógica de cada una de las actividades gracias a la dimensión recreativa. En este sentido, la calidad profesional de sus responsables, de los facilitadores, la capacidad de planificación, la experiencia sistematizada, la coordinación interna, la excelente atención a las personas participantes durante la ejecución de un programa complejo y bien diseñado, coadyuvará en gran medida al nivel de crecimiento institucional y cumplimiento de objetivos del programa.

\subsection{El Campamento Urbano "Vacaciones Felices" en el Museo de los Niños}

El caso del programa de Vacaciones Felices del Museo de los Niños de Costa Rica es un claro ejemplo de un Campamento educativo organizado. Consideramos, según lo planteado en este artículo, que en este programa se promueven los siguientes procesos educativos:

- Aprender haciendo.

- Aprender jugando.

- Aprender por descubrimiento.

- Metodologías educativas participativas.

- Recreación educativa en un contexto patrimonial y propio de un Museo interactivo.

- Trabajo colaborativo y en equipo.

- Aprendizaje vivencial.

- Evaluación de proceso y participativa.

- Integración social y desarrollo de competencias comunicativas.

- Tolerancia y respeto por las diferencias culturales y sociales.

- Integración de actividades que faciliten procesos socio-cognoscitivos, psicomotores y actitudinales.

- Promoción del aprendizaje significativo.

- Transdiciplinaridad: al integrar las ciencias naturales con las sociales y humanas.

En la actualidad, este programa supone la realización de 400 talleres por año totalmente novedosos, respecto a los ofrecidos en el año anterior. Los grupos de edad han cambiado en el tiempo. Actualmente, se trabaja con cuatro grupos: niños y niñas de 3 años y 6 meses a 4 años y 11 meses; de 5 a 6 años; de 7 a 8 años; de 9 a 10 años y de 11 a 13 años. Cada grupo Volumen 11, Número 1, Año 2011, ISSN 1409-4703 
cuenta con un programa de actividades diferente y adecuado a sus intereses y habilidades, pero con temas similares.

En los ya más de 10 años de existencia del programa, fundado en 1999, han participado más de 4 mil niños y niñas. Cada año se planifica y ejecuta el programa en vacaciones de medio periodo (julio) y de inicio de año (enero), con base en un eje temático permite la incorporación de actividades educativas y recreativas específicas al tema.

Uno de los retos centrales de Vacaciones Felices será incorporar la dimensión tecnológica multi-usuario que supone trabajo colaborativo para ser parte de comunidades de aprendizaje en línea, donde el mundo virtual facilitado por la Internet y los software educativos tengan un papel pedagógicamente orientado, pero con una dimensión social protagonista. El tipo de actividades que se han implementado hasta el momento están en el plano del mono-usuario (trabajo individual con la computadora).

La nueva modalidad de multi-usuario, propia de la Web 2.0, permite la construcción del conocimiento de manera compartida lo cual es uno de los intereses del Campamento como programa didáctico: el aprendizaje en interacción social.

Los campamentos urbanos didácticos en los museos necesitan considerar las fases de planificación, la organización, la ejecución y la evaluación de los procesos administrativos y educacionales, en función de las características y de las necesidades de aprendizaje de las poblaciones que atienden, en consideración, de los fundamentos teóricos de la educación no formal aplicada a una didáctica alternativa y vivencial.

\section{REFERENCIAS}

Arco, José y Fernández, Francisco. (2007). Evaluación de programas en educación no formal. En: Torres y Pareja La educación no formal y diferenciada: fundamentos didácticos y organizativos (pp. 667-690). España: Editorial CCS.

Asociación de Scouts de Canadá. (1987) Juegos al aire libre. España: Editorial Martínez Roca. S.A.

Castilla, María Teresa. (2007). Las comunidades educadoras. Propuestas organizativas, metodológicas y de gestión. En: Torres y Pareja, La educación no formal y diferenciada: fundamentos didácticos y organizativos (pp.289-321). España: Editorial CCS.

Danilov, Víctor J. (2005). Women and Museums: A Comprehensive Guide. England: Altamira Press. 
Falk, John y Dierking, Lynn. (2000). Learning from Museums: Visitor Experiences and the Making of Meaning. England: Altamira Press.

Fermoso, Paciano. (1994). Pedagogía social: Fundamentación científica. España: Editorial Herder.

Gamboa de Vitelleschi, Susana. (1995). Aprender jugando desde las actitudes sociales. Argentina: Segunda Edición. Bonum

Hailey, Charlie (2009) Camps: A Guide To 21ST Century Space. Massachusetts Institute of Technology. Library Of Congress Cataloging-in-Publication Data. España.

Incarbone, Oscar y Guinguis, Hugo (2006) Actividades recreativas, juegos, campamentos, bailes y canciones. Argentina: Edit. Stadium S.R.L.

Katz, Phillis (Ed.). (2001). Community Connections For Science Education: History and Theory You Can Use. EEUU: Lithographic.

Lorenzo, Manuel. (2007). El liderazgo en las organizaciones educativas no formales. En: Torres y Pareja, La educación no formal y diferenciada: fundamentos didácticos y organizativos (pp. 105-122). España: Editorial CCS.

Luján, Manuel. (2010) La administración de la educación no formal aplicada a las organizaciones sociales: aproximaciones teórico prácticas. Educación: Revista de la Universidad de Costa Rica, 34 (1). Disponible en: www.revista-educación.ucr.ac.cr

Onrubia, Javier, Colomina, Rosa y Engel, Anna. (2009). Los entornos virtuales de aprendizaje basados en el trabajo en grupo y el aprendizaje colaborativo. En: César Coll y Carles Monereo (Eds.), Psicología de la educación virtual (pp. 233-254). España: Ediciones Morarta.

Otero, Herminio e Iglesias, Julio. (1991). Campamento Urbano: Una propuesta para el tiempo libre en la ciudad. España: Editorial CCS.

Parcerisa, Artur. (2007). Didáctica en la educación social: enseñar y aprender fuera de la escuela (6ta ed.). España: Editorial GRAO.

Paris, Leslie. (2008). Children's Nature: The Rise of The American Summer Camp. New York, EEUU: New York University Press.

Parker, Philip. (2008). Gunnery: Webster's Quotations, Facts, Phrases. Estados Unidos de Norteamérica: ICON Group International, Inc.

Pastor, M. Inmaculada. (2004). Pedagogía Museística: Nuevas perspectivas y tendencias actuales. España: Ariel Patrimonio.

Rodríguez, Keilyn. (2005). Aporte de la nueva museología española al Museo de los Niños de Costa Rica. Revista Electrónica Actualidades Investigativas en Educación, 5 (2). 
Universidad de Costa Rica. Instituto de Investigación en Educación. Disponible en: http://revista.inie.ucr.ac.cr/articulos/2-2005/archivos/museo.pdf

Rodríguez, Keilyn y Vargas, Vanessa. (2009). Análisis del experimento como recurso didáctico en talleres de ciencias: el caso del Museo de los Niños de Costa Rica. Revista Electrónica Actualidades Investigativas en Educación, 9 (1). Universidad de Costa Rica. Instituto de Investigación en Educación. Disponible en: http://revista.inie.ucr.ac.cr/articulos/1-2009/pdf/museo.pdf

Ruiz, María Mercedes. (2001). Archipiélago educativo: espacios de formación del sujeto adulto. En: Marcela Gómez y Bertha Orozco, Pensar lo educativo: tejidos conceptuales (pp.75-88). Plaza y Valdéz editores. Cuadernos de construcción conceptual en educación (3) México.

Santiuste, Roberto, Velázquez, Enrique y Villalobos, Juan. (1999). Juegos en el medio natural. España: Pila teleña.

Thompson Learning Corporation. (2009). Summer opportunities for Kids and Teenagers (26a. ed.). EEUU: Thompson Peterson's.

Vigo, Manuel. (2005). Manual para dirigentes de Campamentos organizados (3ª reimp.). Argentina: Editorial Stadium Waichman.

Waichman, Pablo. (2008). Tiempo libre y recreación: Un desafío pedagógico. España: Editorial CCS.

Watts, Linda. (2007). Encyclopedia of American Folklore. Facts On File, Inc. Library Of Congress Cataloging-in-Publication Data. Estados Unidos de Norteamérica. 